\title{
A Time-Overlapping Multiplex VLC System for End-Edge Data Transmission
}

\author{
Tingting Fu $\mathbb{D},{ }^{1}$ Huanghong Zhu $\mathbb{D},{ }^{1}$ Han Hai $\mathbb{D},{ }^{2}$ and Haksrun Lao $\mathbb{D}^{3}$ \\ ${ }^{1}$ School of Computer Science and Technology, Hangzhou Dianzi University, Hangzhou, China \\ ${ }^{2}$ College of Information Science and Technology, Donghua University, Shanghai, China \\ ${ }^{3}$ Center of Engineering and Design, Chong Cheng Chinese School, Phnom Penh, Cambodia \\ Correspondence should be addressed to Haksrun Lao; haksrunlao@hotmail.com
}

Received 5 March 2021; Revised 11 June 2021; Accepted 5 July 2021; Published 10 August 2021

Academic Editor: Mohammad R. Khosravi

Copyright (C) 2021 Tingting Fu et al. This is an open access article distributed under the Creative Commons Attribution License, which permits unrestricted use, distribution, and reproduction in any medium, provided the original work is properly cited.

Communication is one of the most important foundations in the Internet of Things. Although some cutting-edge technologies, such as $5 \mathrm{G}$, have greatly empowered edge computing, electromagnetic interference and pollution make them impracticable in many environments. The visible light communication (VLC) is a new type of wireless communication technology with appealing benefits not presented in radio communications. VLC allows a lamp or other light source to not only serve as illumination but also simultaneously transmit data. Although traditional VLC multiplexing technologies have been able to achieve a high-speed data transmission rate, they require all receivers to use the same modulation means. In many scenarios, various-type receivers coexist; it is costly to incorporate multiple senders to implement adaptive content distribution in ondemand services. In this paper, we propose a new type of VLC multiplexing system, which realizes end-edge data transmission through pulse position modulation (PPM), pulse width modulation (PWM), and pulse amplitude modulation (PAM) simultaneously. Therefore, one edge server can serve multiple types of end-users without interference. In order to evaluate the performance of the system, we conduct experiments with different settings of communication distance, communication angle, and different environmental light conditions. For three modulations, the proposed system can achieve a transmission speed three times as that for a single modulation, and reach the accuracy rate of up to $99 \%$ within the proper communication range.

\section{Introduction}

Along with the incredible growth of mobile devices in the Internet of Things (IoT) and the explosion of demands for energy and resource-hungry applications, such as online shopping [1], video streaming [2], data processing [3], data sharing [4], and space-air-ground communications [5], the multiaccess edge computing demonstrates the possibility to provide infrastructure, platform, and software as a service for end-users from edge servers with a fixed or wireless network connection [6]. In many cases, an edge server may need to serve multiple end-users simultaneously, where congestion and latency could be very high due to the spectrum crunch problem of wireless communications. Only extending spectrum resources cannot solve the end-edge data transmission problem effectively [7]. Therefore, to reduce the wireless transmission latency between end-users and edge servers, future wireless communications with different radio access technologies, transmission backhauls, and network slices are evaluated in the emerging edge computing paradigm [8].

Different from the emerging RF radio communication system which needs complex signal processing and spectrum sensing means [9], visible light communication (VLC) uses low-cost, energy-saving, and efficient light-emitting diode (LED) to encode the data into high-frequency changes of light intensity that cannot be sensed by human eyes [10]. Various optical sensors (photodiode) can demodulate data by monitoring the change of light intensity. In many cases, the off-the-shelf equipment can be used to realize data communication on the basis of lighting [11]. VLC works in the unregulated spectrum range, and the bandwidth is $10^{4}$ times of the RF [12]. Furthermore, it is free of electromagnetic interference and pollution; therefore, it can be applied in the electromagnetism-sensitive environment, such as inside 
airplanes, hospitals, and scientific machineries. Another important advantage of VLC is being able to provide better security and privacy in some scenarios since light cannot penetrate walls and requires line-of-sight contact with the receiver [13]. For these reasons, VLC can bring communication capabilities between end-users and edge servers, which is characterized by low latency, low cost, good scalability, and privacy protection.

One-to-many communication instances are very common in the edge computing, such as user-specific task services and personalized information deliveries [14]. In such a scenario, multiplexing, which refers to the use of a transmission medium to achieve multichannel signal communication such as MIMO [15] (multi-input and multioutput), can improve the utilization of the link and achieve Burst-Mode communications [16]. In VLC systems, common multiplexing technologies include WDM (Wavelength Division Multiplexing), SDM (Space Division Multiplexing), TDM (Time Division Multiplexing), and FDM (Frequency Division Multiplexing) [17]. WDM and SDM need to use multiple LEDs as transmitters to form a MIMO system. TDM will bring down the transmission rate under the same hardware conditions. FDM is much complex to achieve, and the hardware equipment is more expensive, which is not suitable for simple text data transmission. The transmission rate of these highly complicated VLC devices can reach Gbps, but so far, none of them has been put into use. Therefore, our goal is to use single LED and existing low-cost hardware to design a simple VLC multiplex data communication system and improve the system transmission capacity, where multiple end-users share a communication link.

In this paper, we propose a multiplex data transmission system based on pulse position modulation (PPM) [18], pulse width modulation (PWM) [19], and pulse amplitude modulation (PAM) [20]. The key idea is to use these three modulation methods to encode three groups of data, respectively, then combine three signals into one signal, and send the data stream through a single LED to three categories of end-users simultaneously. Each end-user uses a different demodulation rule to get its dedicated data information, with time-overlapping. This is very useful in many scenarios, especially in a dynamic environment, where end-users come and go frequently. They may expect for different message contents in terms of the group they belong to. By combining multiple modulation methods into one signal, the sender can broadcast different contents to different end-users at one time, without interference.

For instance, the proposed VLC system can be deployed in a vehicular environment where mobility is the main challenge in vehicular edge computing [21,22]. The free space in the frequency spectrum for all normal communication specifications is very full. Therefore, using radio wireless communication to transfer information may have interference from other wireless signals, which could lead to a traffic accident for vehicles. As can be seen from Figure 1, three vehicles have overlapped communication range (shown in different colors), which is potential to cause conflicts. If personalized information has to be transmitted, the time needs to be divided into small slots. Therefore, it is important to use

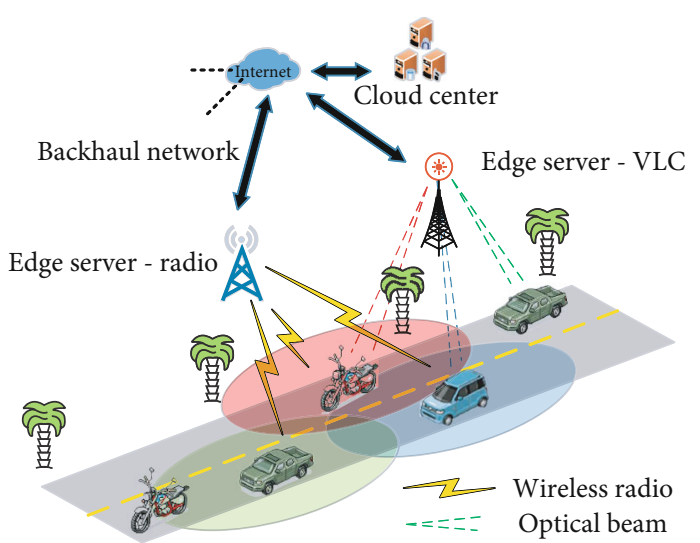

FIGURE 1: Demonstration of multiplexing in edge computing.

new technologies to avoid this happen. Also, the VLC multiplexing system has better and low-cost privacy performance since each group of users uses a different coding scheme. Regarding wireless multiplexing, applying different cipher keys is a common solution. The proposed VLC system is suitable for the problem. Also, it is energy efficient since it is based on LED, and very healthy for humans since the change of lights is undetectable by eyes.

The rest of the paper is organized as follows. We first briefly introduce the latest literatures on data transmission in edge computing and VLC-related research. Then, we draw the big picture of the overall model of the system. After that, the system design, including modulation, demodulation, hardware implementation, and software coding, is described in detail, followed by the experiment results and performance analysis. Finally, we conclude the paper and suggest some open issues for future work.

\section{Related Work}

2.1. Communication in Edge and Industrial Computing. Communication is the key foundation in Edge and Industrial Computing, through which task offloading and data transmission must go with. To enhance communication capability, MIMO is often used in IIoT [23], where multiple antennas are installed. In [24], QoE (Quality of Experience) of the edge-cloud architecture is enhanced by adopting application-level coding (e.g., transcoding, rate control) to match the estimated capacity at the radio downlink. In the Edge Computing-Internet of Vehicles system, two communication models between edge servers and users are considered, i.e., the single-hop communication and the multihop communication [25]. Tasks from user vehicles may be offloaded to $5 \mathrm{G}$ base stations for processing. $5 \mathrm{G}$ can provide fast and reliable data transmission between base stations and cloud servers; however, interference management of wireless communications within a base station deserves further investigations, not to mention security communication problem for drones [26]. In another scenario, where service robots are deployed for healthcare, data is collected from people and sent to the cloud. Robots also act as edge servers to alleviate the burden of the cloud as well as reduce latency [27]. The result of edge computing is exchanged through rapid 
machine-to-machine communication. Low-cost large-scale communication is also very important for mobile edge computing in the maritime Internet of Things, where the channel allocation and power allocation problems are two major means to optimize the offloading policy [28]. With the rapid growth of federated learning in the end-edge-cloud orchestra, in most cases, it may suffer from a large number of rounds to convergence, which leads to high communication costs. Some work [29] proposes novel compression techniques called FedAvg to produce communication-efficient.

2.2. VLC System and Multiplexing. The early research of VLC was initiated by a team led by Nakagawa of Japan. They envisioned the combination of VLC and PLC to provide indoor network communication [30] and firstly studied the environmental characteristics of VLC indoor propagation [10]. Since then, increasing communication rate has become a major research direction of VLC, including filtering yellow light [31], designing complex modulation schemes (for example, CSK [32]), and using multiple inputs and multiple outputs to realize parallel data stream transmission [33]. At present, the fastest VLC communication system can reach a speed of $10 \mathrm{Gbps}$.

Professor Chi Nan of the Fudan University introduced the use of several traditional multiplexing technologies in VLC and verified the feasibility of multiplexing technology in improving the transmission capacity of the VLC system [12]. The multiplexing technologies that can be used include wavelength division multiplexing, space division multiplexing, polarization multiplexing, and frequency division multiplexing [12]. Wavelength division multiplexing (WDM) refers to using visible light of different wavelengths as carrier waves to modulate signals. For RGB-LED, red, green, and blue visible light of different wavelengths can be used to modulate different signals, respectively. Space division multiplexing uses multiple transmitters to send data and multiple receivers to receive data at the same time, to realize parallel transmission of space multiplexing. Polarization multiplexing is to modulate the signal to the linearly polarized light in different directions by using the visible polarizer and to carry out multichannel parallel transmission. Frequency division multiplexing (FDM) is to realize multiclient parallel transmission in frequency by using subcarrier modulation signals with different center frequencies of LED. The transmission rate of these traditional multiplexing technologies can be up to gigabits per second.

At present, there are a bunch of researches on VLC modulation methods. We can divide it into two categories: (1) single carrier modulation mechanism [34], such as pulse width modulation (PWM), pulse amplitude modulation (PAM), and pulse position modulation (PPM); (2) multicarrier modulation mechanism [35], which requires more complex hardware support, such as orthogonal frequency division multiplexing (OFDM). Different from existing research, we developed a new VLC multiplexing system for low data rate and cost-sensitive applications.

\section{System Model}

The overall diagram of the core time-overlapping multiplex VLC system is shown in Figure 2. For different applications,
LEDs can vary in power, and the driver circuit should be designed correspondingly. The proposed communication system can support up to three categories of end-users in the same direction. Each category can have multiple endusers. In the figure, each category is represented by one end-user. The edge server is used to transmit the data stream to the agent microcontroller. The microcontroller modulates the data going to be sent, uses the modulated signal to control the MOSFET, and loads it on the LED light source. Under the control of the MOSFET, the LED lights up quickly, and finally, the visible light is carried in the communication channel with data dissemination. At the receiving end, the rapid intensity change of LED will be captured by photodiodes. Each photodiode converts the optical signal containing information into an electrical signal and then uses the microcontroller connected with the photodiode to sample the electrical signal generated by the photodiode. Finally, each end-user will process the sampling data according to its dedicated demodulation rule, restore the original information as it been sent.

\section{System Design}

The VLC multiplexing data transmission system encodes the data of different categories and then sends the data to each end-user simultaneously through the modulation mode of PPM, PWM, and PAM. Each end-user uses different demodulation methods to obtain their data. Each end-user does not interfere with another end-user, nor affects each other, and is not able to extract data belonging to other end-users.

4.1. Channel Model. Let us assume that the proposed visible light communication system is composed of LED transmitters and end-users. Then, the received signal can be considered as in Equation (1) [36]:

$$
y(t)=\sqrt{\rho} H(t) \otimes s(t)+n(t),
$$

where $\rho=\left(r^{2} \bar{P}_{K}^{2}\right) / \sigma^{2}$ denotes the average electrical SNR at each receive unit, $\otimes$ is time convolution, $\bar{P}_{K}^{2}=(1 / K) \sum_{i=1}^{K} P_{K}^{(i)}$ represents the average received optical power, and $r$ denotes the photodiode responsivity. The vector $n(t)$ indicates $K$ -dimensional noise. The noise includes the receiver thermal noise and shot noise due to ambient light. Thus, $n(t)$ can be modeled as independent and identically distributed additive white Gaussian noise with power spectral density $\sigma^{2}=$ $\sigma_{\text {shot }}^{2}+\sigma_{\text {thermal }}^{2}$, where $\sigma_{\text {shot }}^{2}$ is the shot noise variance and $\sigma_{\text {thermal }}^{2}$ is the thermal noise variance [37].

For VLC systems, the direct line-of-sight (LOS) and the nondirect line-of-sight (NLOS) are the two general models. In this paper, we only consider the LOS propagation path since LOS accounts for the most total received optical power at the receiver. Let $\mathbf{H}_{k} \in \mathbb{R}^{1 \times N_{T}}$ denotes the channel matrix between transmitter and receiver (as shown in Equation (2)):

$$
\mathbf{H}_{k}=\left[h_{k 1}, h_{k 2}, \cdots, h_{k N_{T}}\right]
$$

where $h_{k i}$ represents the direct current gain between the $k$-th 


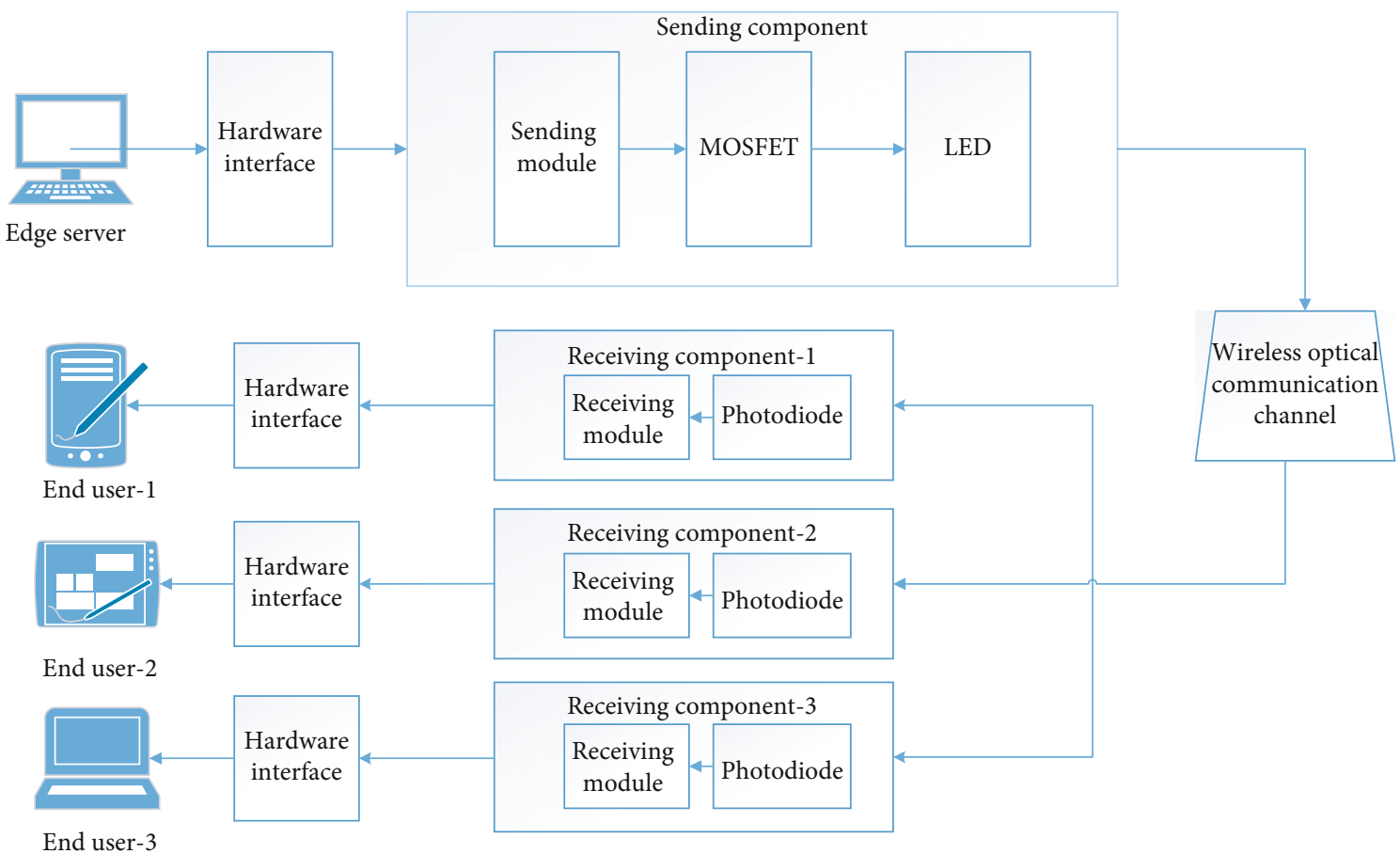

FIGURE 2: Core model of the proposed time-overlapping multiplex VLC system.

end-user and the $i$-th transmitter. In terms of the LOS case, $h_{k i}$ is given by [10] as shown in Equation (3):

$$
h_{k i}= \begin{cases}\frac{A_{r}}{d_{k i}^{2}} L(\phi) T_{s}\left(\psi_{k i}\right) g\left(\psi_{k i}\right) \cos \left(\psi_{k i}\right), & 0 \leq \psi_{k i} \leq \Psi_{c}, \\ 0, & \psi_{k i}>\Psi_{c} .\end{cases}
$$

where $A_{r}$ is the active area of the photodiode and $d_{k i}$ is the distance from the LED to the photodiode. $T_{s}\left(\psi_{k i}\right)$ is the gain of the optical filter and $\psi_{k i}$ denotes the angle of incidence. The optical field of the view of the photodiode can be denoted by $\Psi_{c}$. Finally, $g\left(\psi_{k i}\right)$ denotes the optical concentrator gain.

4.2. Modulation. The PPM is based on the position of the pulse. PPM is to divide the time of a cycle into several time slots of equal intervals, and the transmission of the pulse is done in any one of the slots. According to the corresponding relationship between data and pulse position, the sender chooses to send a pulse signal in a certain time slot to realize data transmission. The design principle of PPM is shown in Figure 3(a). A cycle is divided into two time slots. The first slot has a pulse signal indicating data bit " 1 ," and the second slot has a pulse signal indicating bit " 0. ."

The PWM uses pulse duration to control the LED drive current; thus, it can adjust the brightness. The advantage of the PWM is that it does not suffer from the wavelength shift due to the current variation in the intensity or amplitude modulation-based scheme. In PWM, brightness level with a wide range $(0-100 \%)$ can be achieved by directly adjusting the modulation index. Moreover, the human eyes cannot sense the current switching since the dimming signal fre- quency is usually above $100 \mathrm{~Hz}$. PWM uses different widths of the pulse signal to realize data modulation. The design principle of PWM is shown in Figure 3(b). Different width of the pulse signal in a cycle time represents bit " 1 " and bit " 0 ."

PAM refers to a modulation mode in which the pulse height changes with the encoding. It is a bandwidth efficient scheme since it can improve spectral efficiency. Data is modulated into different amplitudes of the signal pulse. PAM may suffer nonlinearity in LEDs' luminous efficacy due to the modulation schemes employing different intensity levels. Since the light emitted by an LED depends on the input current and temperature, it changes at multiple symbol levels of the PAM along with changes in the drive current. The design principle of PAM is shown in Figure 3(c). The different heights of the pulse signal in a cycle time are used to represent bit " 1 " and bit " 0 ."

The visible light communication system adopts intensity modulation and direct detection mechanism. The power of LED can be expressed as Equation (4):

$$
P_{\text {led }}=V_{\text {led }} * I_{\text {led }} \text {. }
$$

where $V_{\text {led }}$ is the voltage of LED light source and $I_{\text {led }}$ is the current of LED light source. The peak intensity of LED light source is mainly affected by the LED power. Therefore, by using different input voltage, it can generate pulse signals of different heights and, finally, act on the LED to change the peak intensity of the LED light source.

In this paper, we propose to use PPM, PWM, and PAM modulation technologies to implement the VLC multiplexing data transmission system for end-edge data transmission. By modulating the pulse position, pulse width, and pulse amplitude simultaneously, we can send data to three categories of 


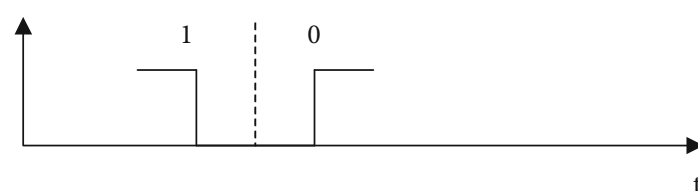

(a) PPM modulation

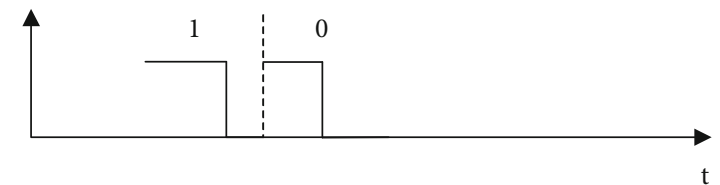

(b) PWM modulation

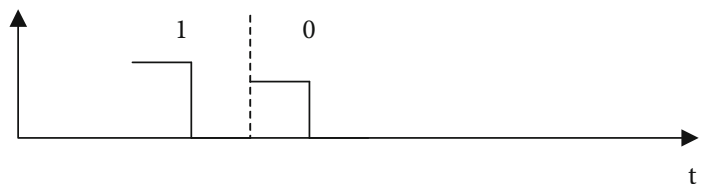

(c) PAM modulation

FIGURE 3: PPM, PWM, and PAM modulation.

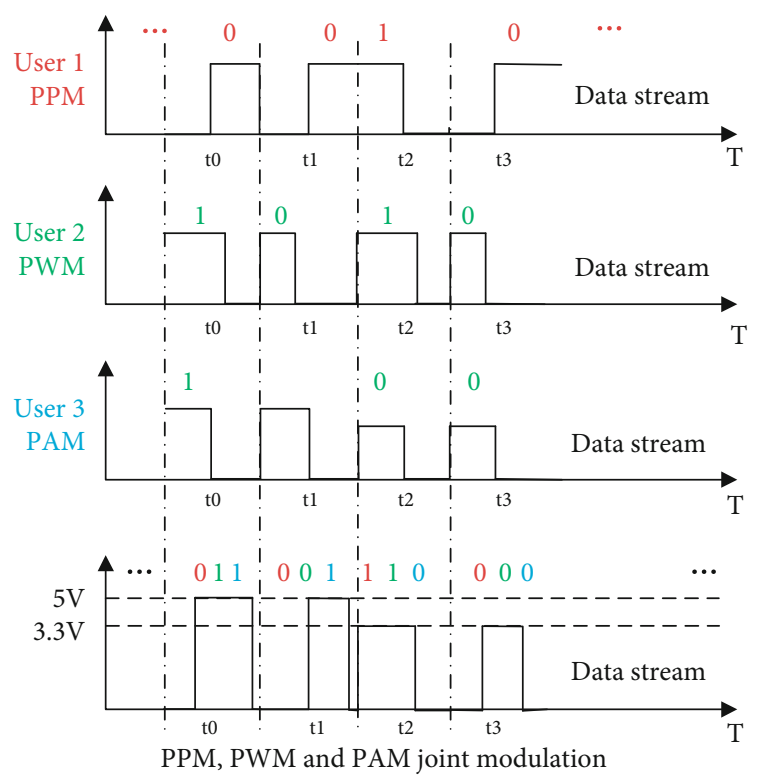

Figure 4: PPM, PWM, and PAM joint modulation signal diagram.

end-users at the same time, for instance, end-user 1 with PPM, end-user 2 with PWM, and end-user 3 with PAM. Suppose that data stream " 0010 " is going to be sent to the enduser 1, data stream " 1010 " is going to be sent to the enduser 2, and data stream " 1100 " is going to be sent to the end-user 3. The modulation design is shown in Figure 4; the server first encodes data into light pulse modulations for each end-user and then integrates them into one signal with changing position, width, and height. The first bits of three users are, respectively " 0 ," " 1 ," and " 1 ." Therefore, the corresponding joint modulation signal starts with a low pulse and ends with a high pulse in the time slot, and the high pulse has a larger width and a higher amplitude. Each end-user can extract the exclusive data from the same signal waveform.

However, in some hybrid system where both $5 \mathrm{~V}$ and $3.3 \mathrm{~V}$ power are mixed, if a previous data starts with a low pulse, ends with a high pulse of $5 \mathrm{~V}$ power supply, and the following data starts with a high pulse of $3.3 \mathrm{~V}$ power supply, ends with a low pulse, there will be a direct conversion from $5 \mathrm{~V}$ to $3.3 \mathrm{~V}$ between two data. The problem is before the $5 \mathrm{~V}$ power supply has been completely turned off, the $3.3 \mathrm{~V}$ power supply has been turned on, so chaotic data will be generated, increasing the difficulty of decoding. Our solution is to check whether a signal starts with a low pulse and ends with a high pulse, immediately add a low pulse delay after the high pulse to ensure that the power supply is completely shut down.

4.3. Demodulation. The receiving end-user continuously senses the intensity of the incident light through the photodiode, processes the collected intensity value, and restores the original data. For each end-user, first of all, we need to locate the rising edge and the falling edge of each periodic optical pulse [13]. We can find the local maximum value to locate the edge of the optical pulse by calculating the first derivative $I^{\prime}(x)$ of the intensity value as shown in Equation (5):

$$
I^{\prime}(x)=-\frac{1}{2} \cdot I(x-1)+0 \cdot I(x)+\frac{1}{2} \cdot I(x+1)
$$

where $I(x)$ is the intensity perceived by the photodiode.

Each end-user applies different demodulation methods. For end-user 1, the data is decoded by the PPM principle to find the relative positions of high pulse and low pulse in the cycle, and the original data information is restored according to different positions of the pulse. For end-user 2, data is decoded by the PWM principle, the high pulse width is calculated by a rising edge and a falling edge of the pulse, and original data information is restored according to different width values. For end-user 3, we decode the data by the PAM principle, find the maximum light intensity detected in each cycle, and compare it with the peak intensity of LED when connecting to $5 \mathrm{~V}$ power supply and $3.3 \mathrm{~V}$ power supply, respectively, and finally, restore the original data information. However, the peak intensity of LED decays with the square of the distance between the LED and the photodiode, and the different distance between the LED and the photodiode will produce a difference in the peak intensity detected by the photodiode. Therefore, our solution is to add two bits of light intensity calibration before sending each data packet, where the first bit uses a $5 \mathrm{~V}$ power supply, and the second one uses a $3.3 \mathrm{~V}$ power supply (as shown in Figure 5). Finally, when restoring the data information, the peak intensity of the data bits only needs to be compared with that of the first two calibration bits. 


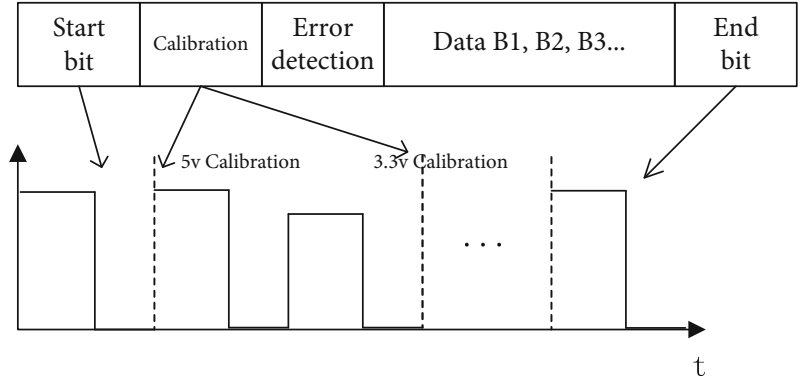

Figure 5: Design of calibration in PAM model.
In many practical data transmission processes, the length of data stream to be sent to each end-user is different. A binary bit with no meaning could be recognized as data for end-users improperly. Therefore, we add a stop bit at the end of each data stream, according to which the end-user judge whether to continue to accept the data. Bit " 1 " means continuing to receive and bit " 0 " means stopping receiving.

4.4. Error Detection and Correction. In this paper, we build an Arduino UNO-based experiment platform as the validation system, due to the limited sampling rate; the pulse width corresponding to a bit may be inaccurate during decoding, resulting in decoding error of that bit in the process of data transmission. Moreover, consider that many VLC systems only need a one-way transmission, where it is not possible to get the receiver's feedback information; hence, we use FEC (Forward Error Correction) to detect and correct errors to achieve reliable transmission. Hamming code is a type of linear block code, which has been widely used in the telecommunication system. It can correct random errors and burst errors. In our design, Hamming code is adopted to add redundant bits in data to realize error detection and correction. It organizes data bits into groups, determines whether there are any errors in the group through parity check, in terms of the principle of odd or even matching. Concretely, the total length of the data frame is 14 bits, which includes 2 calibration bits, 8 data bits, and 4 parity check bits. Hamming code can only recover 1 bit error. If more than 1 data bits flip during the transmission, Hamming code will not work. However, during the experiment, we find that very few bit errors occur and the vast majority of them is 1 bit error, providing that the communication range is within the proper distance. The reason we chose Hamming code is that it is very simple and easy to implement. It also puts little cost over transmission.

4.5. Design of the Validation System. The evaluation system includes a sending module and a receiving module. The sending module mainly includes one microcontroller, one MOSFET Driver Module, and one LED light source. The receiving end mainly includes one microcontroller, one resistor, and one photodiode. The specifications of the LED light source and the photodiode used in the experiment are shown in Table 1.

On the transmitter side, the microcontroller is Arduino UNO [38], with a clock frequency of $16 \mathrm{MHz}$, which can generate microsecond level pulses. Two pins (pin 9 and pin 10)
TABLE 1: Component parameters.

\begin{tabular}{lc}
\hline Component & Parameter \\
\hline & Voltage range: $3.3-5 \mathrm{~V}$ \\
Parameters of LED & Power: $\mathrm{W}$ \\
& Angle of view: $90^{\circ}$ \\
& Intensity: $200-300 \mathrm{~lx}$ \\
& Wavelength: $300-760 \mathrm{~nm}$ \\
\hline & Photocurrent: $10 \mu \mathrm{A}$ \\
Parameters of Photodiode & Rise/fall time: $12 \mathrm{~ns}$ \\
& Peak wavelength: $850 \mathrm{~nm}$ \\
\hline
\end{tabular}

of the microcontroller are connected with two p-type MOSFET driver modules. The two p-type MOSFET modules are, respectively, connected with $3.3 \mathrm{~V}$ and $5 \mathrm{~V}$ voltage, and one LED light source. Through controlling the two MOSFET drivers, the LED can be quickly switched on and off. The control circuit of the transmitter is shown in Figure 6.

The receiver also uses Arduino UNO as the microcontroller to connect the photodiode at pin A0 and sample the data of the photodiode with a sampling frequency of $50 \mathrm{kHz}$. It can map the voltage collected from the photodiode to a value from 0 until 1023. A $1 \mathrm{~m} \Omega$ resistor is connected at the receiving end to improve the gain of the photodiode. The control circuit of the receiving end is shown in Figure 7.

\section{Results and Analysis}

We test the proposed time-overlapping multiplex VLC system from two aspects: (1) system function test: whether the transmitter (edge server) and the receiver (end-user) can reliably conduct one to three communications; (2) system performance test: test the speed and accuracy of the system under different link distance, different perspective, and different ambient light conditions. Due to a limited budget, we only build a minimal system that can fulfill the evaluation purpose. The prototype of the experiment system is shown in Figure 8. One LED controlled by the Arduino board acts as the edge server (Figure 8(a)) while three Arduino boards with photodiodes are end-users (each of which uses PPM, PWM, and PAM, respectively, Figure 8(b)). By default, the experiment is carried out indoor and under the condition of turning on the fluorescent lamp, the photodiode is directly below the LED, with a height of $13 \mathrm{~cm}$. The period of transmitting signal is set as $220 \mu \mathrm{s}$, and the pulse width modulated high pulse width is $140 \mu$ s and $100 \mu$ s, respectively.

5.1. System Function Experiment. The system function test experiment is carried out by sending text data to receivers. The text data needed to be sent to end-users is input from the console. In the test, we arbitrarily select the characters "a," " 6 ," and “*," corresponding to end-user 1 , end-user 2 , and end-user 3, respectively. The received data printed on the end-user console validates that the letter, the number, and the symbol can be correctly transmitted.

In order to ensure that the system can work normally, we draw the pulse shape diagram of the transmitter and the receiver (as shown in Figure 9). The pulse shape diagram 


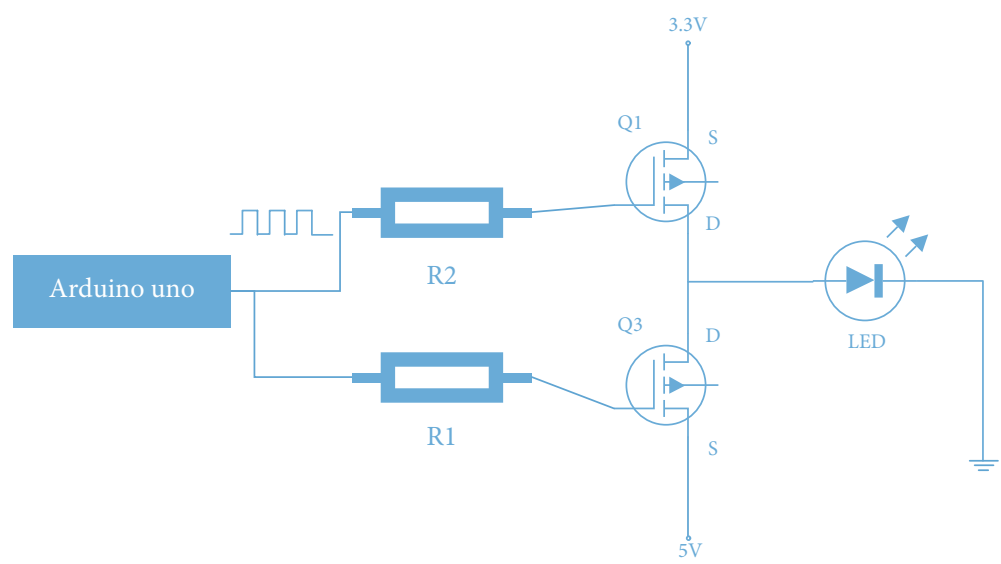

FIgURE 6: Hardware design of the transmitter.

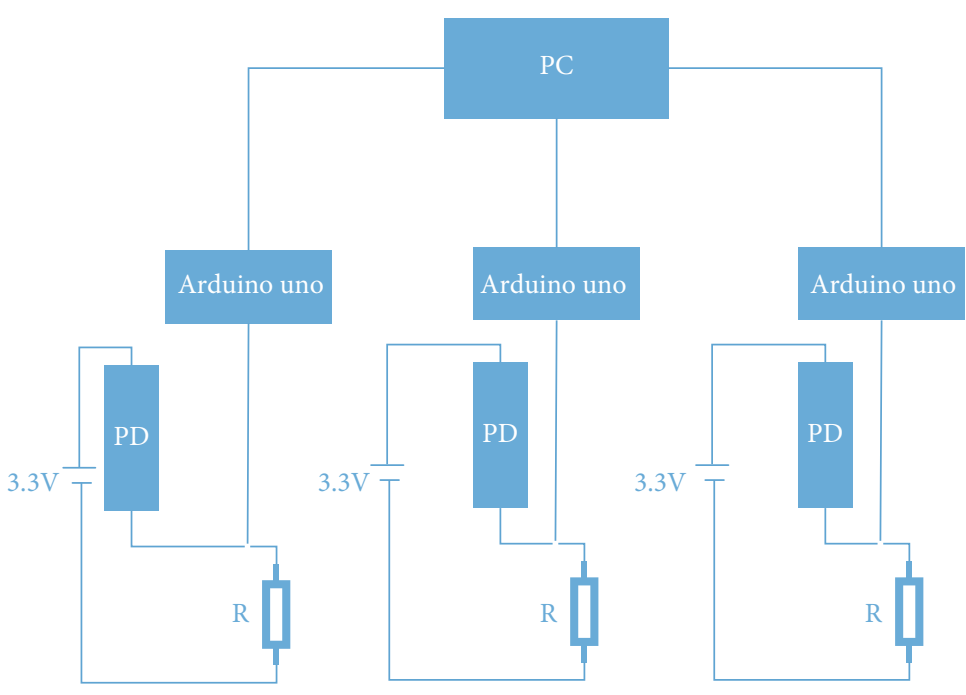

FIgURE 7: Hardware design of receivers.

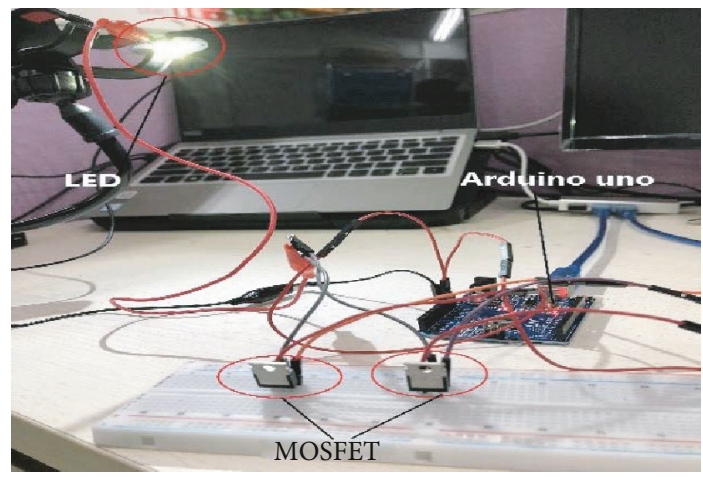

(a) Transmitter subsystem

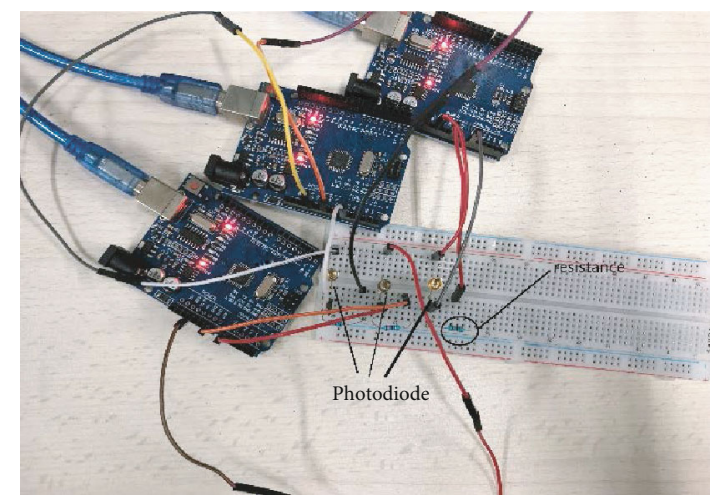

(b) Receiver subsystem

Figure 8: Experiment system implementation.

shows that the receiver can correctly receive the signal from the transmitter.

5.2. System Performance Experiment. By testing the throughput and accuracy of the VLC multiplexing system, we inves- tigate the influence of communication channel parameters and environmental factors on the system performance. Throughput is the number of bits received correctly per second, and accuracy is the ratio of the number of bits received correctly to all bits sent. In each experiment, the sender sends 


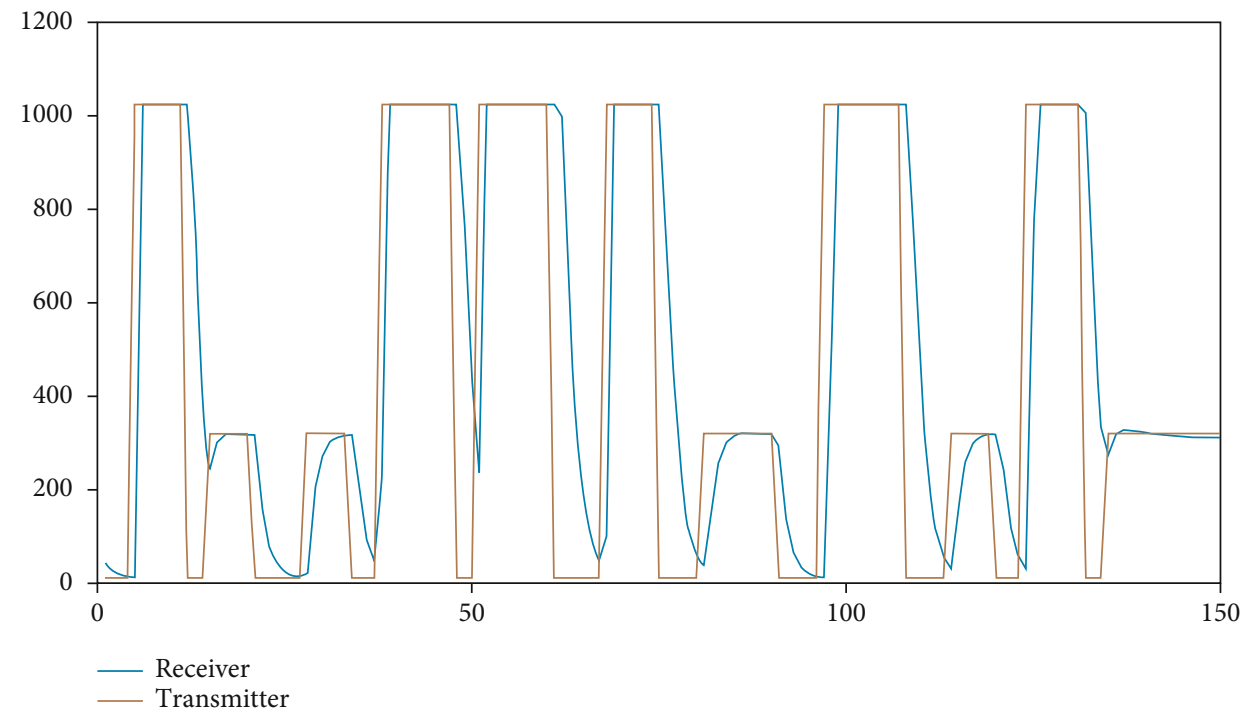

Figure 9: Pulse signal diagram of the transmitter and receiver.

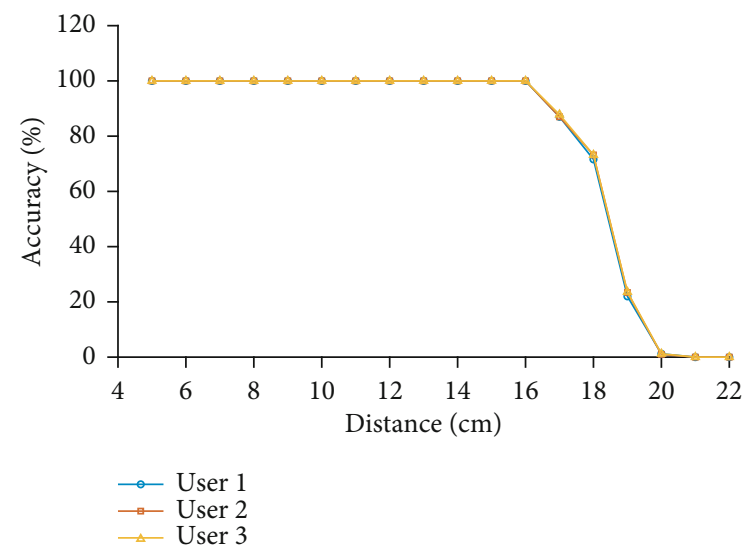

(a) Influence of communication distance on system accuracy

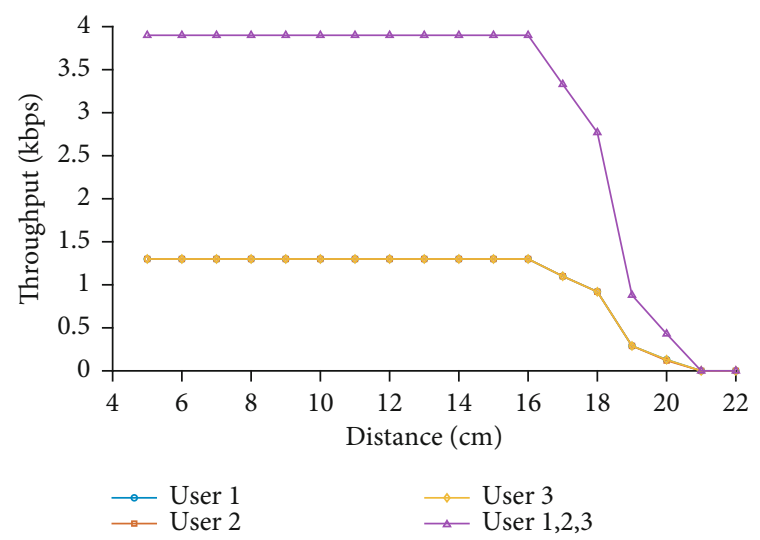

(b) Influence of communication distance on throughput

FIGURE 10: Influence of communication distance on the system.

512 random bits of data to each end-user at the same time. We repeat the experiment ten times to calculate the average throughput and accuracy.

5.2.1. The Influence of Link Distance on the System. The link distance between LED and photodiode is one of the important parameters that affect the accuracy of data transmission. We adjust the distance from the LED to the photodiode from $5 \mathrm{~cm}$ to $22 \mathrm{~cm}$ and keep the LED directly below the photodiode. The experimental results are shown in Figures 10(a) and 10 (b). We can see that the system can support a $16 \mathrm{~cm}$ communication distance, and the throughput of each end-user is about $1.29 \mathrm{Kbps}$; the total throughput is $3.87 \mathrm{kbps}$. As long as the distance between the sender and the receiver is within the communication range, the data can be transmitted reliably. When the communication distance is more than $20 \mathrm{~cm}$, its data transmission accuracy becomes $0 \%$. We can see that no matter what kind of modulation means are used, the curves are highly coincident. This is because once the communication range is over length, the current generated by the photodiode induced light intensity changes very little, which makes Arduino unable to sample it.

Due to the low power of the LED $(5 \mathrm{~W})$ in the validation system, the valid distance is around $16 \mathrm{~cm}$. The power used in related work is around $20 \mathrm{~W}$ to $30 \mathrm{~W}$. Therefore, the effective distance can be extended by applying a LED with larger power. Under the same condition, it is expected that our multiplexing system can achieve the same distance performance as in the related work (up to 100 meters). Furthermore, the distance can be extended by adding an optical lens at the receiving end. Finally, increasing the frequency of the microcontroller (in our system, Arduino UNO is a low-end microcontroller) can directly improve the data rate to a much higher level (15 Gbps in a report).

\subsubsection{The Influence of Different Ambient Light Conditions on} the System. To test the robustness of the system, we carry out experiments at 2:00 pm and 7:00 pm, respectively, under different ambient light conditions and turn on the fluorescent lamp in both cases. The experimental results of end-user 1 


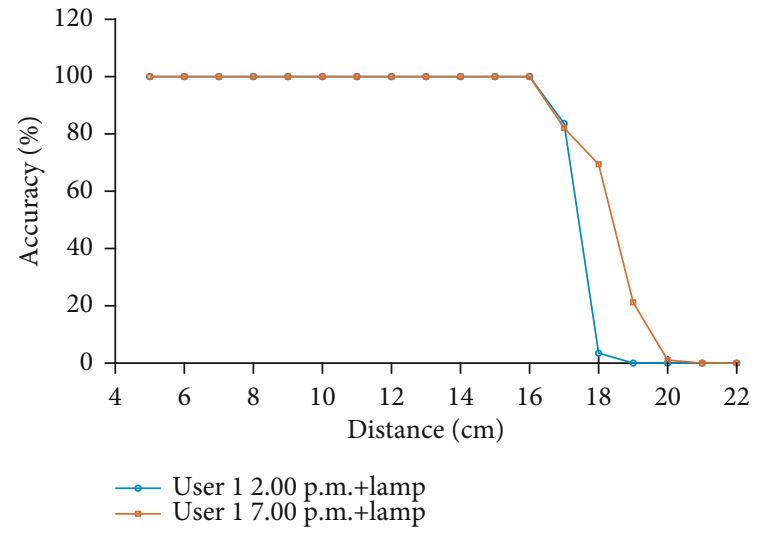

FIGURE 11: Influence of ambient light conditions on the system.

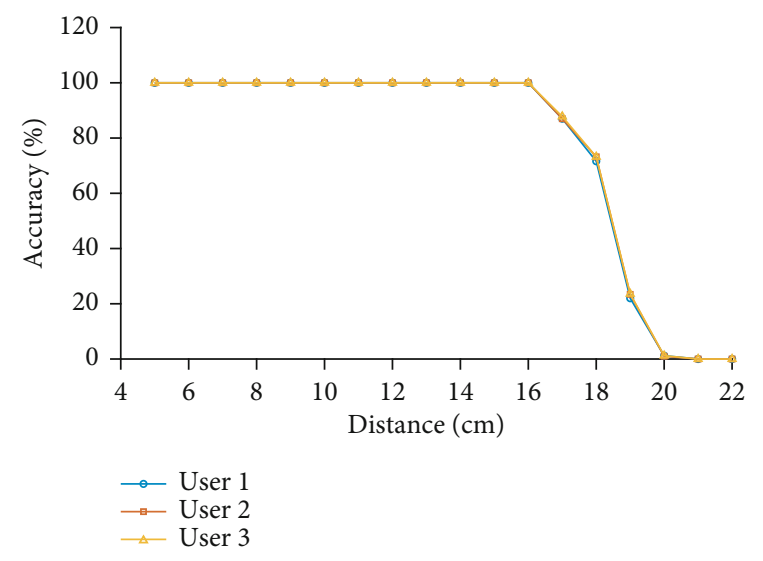

FIGURE 12: Influence of different angle of view on the system.

under different ambient light conditions are shown in Figure 11 . We can see that at two o'clock in the afternoon, when the ambient illumination is high, the maximum supported communication distance of the system is $16 \mathrm{~cm}$, which is the same as the experiment at 7:00 pm. When the communication distance is more than $18 \mathrm{~cm}$, the effect of the ambient light starts to appear; however, the result got at 2:00 pm has little improvement. Therefore, we can say that the system has a strong tolerance to ambient light.

5.2.3. The Influence of Angle of View on the System. The angle of view is also another important parameter to measure the system. It refers to the angle between the LED and photodiode line and the LED normal. We adjust the viewing angle from $0^{\circ}$ to and $35^{\circ}$. The experiment is carried out at 7:00 $\mathrm{pm}$ and the distance between LED and photodiode is $13 \mathrm{~cm}$. The experimental results are shown in Figure 12. We can see that the angle of view supported by the system is about $40^{\circ}\left( \pm 20^{\circ}\right)$.

5.2.4. The Performance Comparison with PWM Multiplexing. As most of recent work is moved to use multiple-input multiple-output (MIMO) hybrid multiplexing in VLC due to achievable very high data rate, there are few researches insisting on using single LED. However, not all applications need such high data rate. They may concern more on deploy-

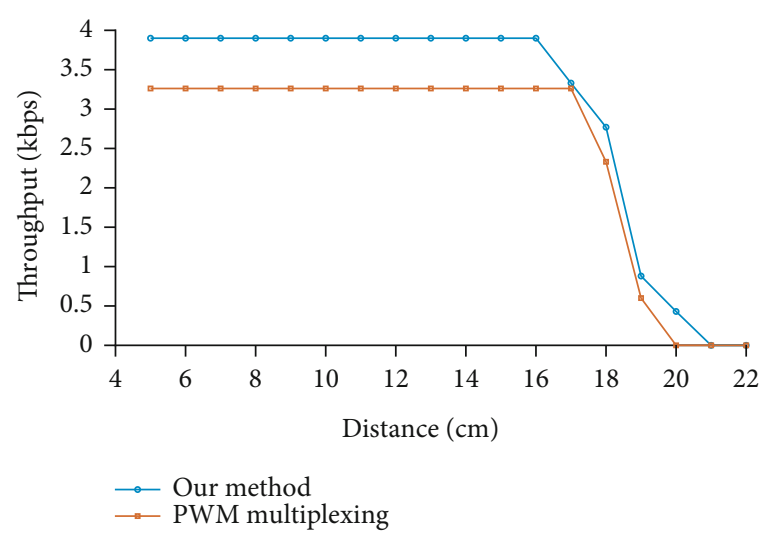

FIGURE 13: The performance comparison with PWM multiplexing.

ing cost, where multiplexing with a single LED is very useful. To our best knowledge, there is no such work combining PPM, PWM, and PAM as we do, therefore, we compare our method with a traditional multiplexing method only using pulse width modulation (PWM).

As shown in Figure 13, the total throughput of PWM multiplexing with three users is around $3.27 \mathrm{Kbps}$, which is only $84 \%$ of the throughput of our method. Furthermore, with the increase of the number of users, the number of pulse width will also increase in an exponential manner, which will greatly lower per user throughput.

5.2.5. The Test on Vast Transmission. Since large data files, such as images and videos, are very common in data transmission, to test the robustness of the proposed system, we have carried out image transmission experiments. We choose the most famous image "Lena" as the test object. First, we convert the standard ".tif" file into ".eps" format, which size is around $1560 \mathrm{~KB}$. Second, we send the image using PPM, PWM, and PAM modulation simultaneously. In this step, we read the eps file byte by byte and apply Hamming code before transmission. The actual size of each image transmitted is $2730 \mathrm{~KB}$. Third, we analyze the difference of the original image and the received image. Since the data rate is about $1.29 \mathrm{Kbps}$, it costs around 2100 seconds (35 minutes) to finish one transmission. We repeat the experiment for 100 times and find that, in $93 \%$ of the cases, we get the equal images. In the remaining 7 cases, the differences between two images are less than 2 bytes in 5 results. Most of bit errors have already been corrected by Hamming code. As shown in Figure 14, (a) is the original image and (b) is the received image. The differences between them are 41 bytes (possessing $0.00256 \%$ of total bits), which is the worst case in the 100 time experiments. However, the human eyes still cannot tell the difference between them.

Therefore, the proposed system can handle most of practical application scenarios with reasonable cost. However, if the hard robustness data transmission needs to be guaranteed, either a two-way communication means or much complex Forward Error Correction Encoding must be adopted, which violates the design purpose of this system. 


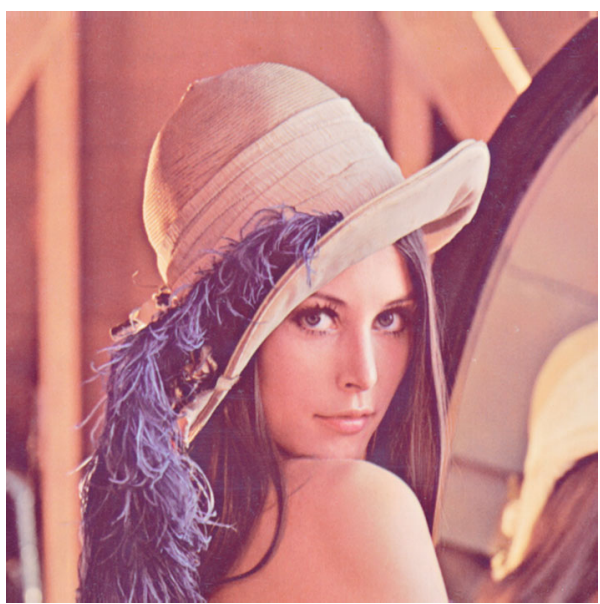

(a) Lena-original

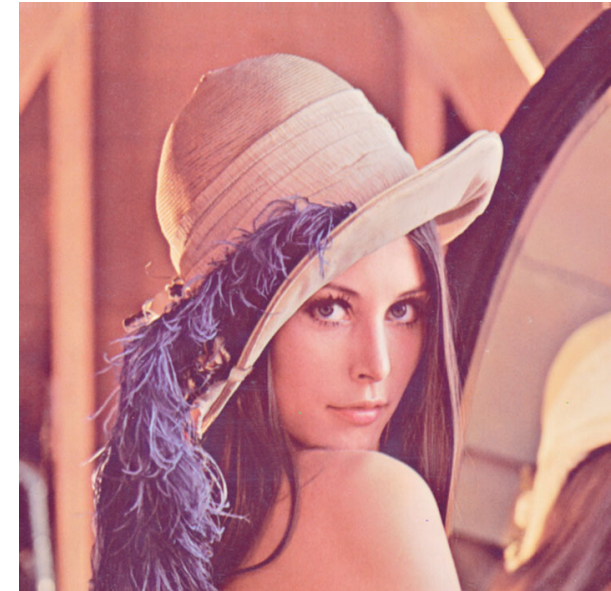

(b) Lena-received

FIGURE 14: Image transmission result.

5.3. Potential Applications. Besides end-edge data transmission, the proposed system can be applied in many IoT applications, such as underwater communication, medical treatment, and indoor positioning.

Because of the high attenuation rate of electromagnetic wave, it is difficult to use radio waves for underwater wireless communication. Compared with traditional underwater sonar communication, visible light optical communication has higher directivity and confidentiality. Taiyo Yuden and Toyo Electric Co jointly developed a high-speed underwater wireless communication device based on visible light. Using the general blue LED with low attenuation rate in water, the maximum communication speed of $50 \mathrm{Mbps}$ is achieved.

For hospital and medical field, visible light communication is considered to be the most suitable wireless communication mode since it does not cause electromagnetic interference to electronic equipment. Whether it is to realize the dynamic monitoring of patients or the communication between medical devices, VLC provides a safe and reliable way of information transmission. The hospital is likely to be the first large-scale popularization of VLC system.

The proposed VLC system uses LED as transmitter, which are currently being installed in most buildings, for instance, large shopping malls, office, and classroom. Since the global positioning system (GPS) signals are difficult to pass through building walls, the VLC-based indoor positioning system can be a good substitute. It estimates location by the geometric properties of triangles. Specifically, this technique uses difference reference points to get target position, where the reference points are LEDs, and target is the optical receiver. Comparing to radio frequency-based indoor positioning system, VLC is electromagnetic interference free and can achieve a good accuracy.

\section{Conclusion}

In this paper, we proposed a new VLC system to use PPM, PWM, and PAM modulation technology to realize the VLC multiplexing communication system for end-edge data transmission. We designed the modulation methods and solved the problem of the nonfixed length of individual information. Also, a delay was added to fit the mixed voltage system. We used a low-cost hardware solution, in which an offthe-shelf LED and photodiode are adopted to build the preliminary validation prototype. The proposed VLC system successfully completed a one-to-three communication. The communication rate of a single client can reach $1.29 \mathrm{Kbps}$, the communication distance can reach $16 \mathrm{~cm}$, and the accuracy is near $99 \%$.

Future research issues include further improving communication range and data rate and efficient two-ways VLC communications. With the more powerful LEDs and microcontrollers, the range and the rate can be easily extended to a level that has the potential to be used in many scenarios, such as underwater communication, medical treatment, and indoor positioning to avoid radio communication interference and also improve safety.

\section{Data Availability}

Data is available on request; please contact the corresponding author Haksrun Lao (haksrunlao@hotmail.com).

\section{Conflicts of Interest}

The authors declare that there is no conflict of interest regarding the publication of this paper.

\section{Authors' Contributions}

This is a joint work with Mr. Huanghong Zhu. Prof. Tingting $\mathrm{Fu}$ was the supervisor of Mr. Zhu and Mr. Lao when Haksrun Lao was an undergraduate student at Hangzhou Dianzi University. Dr. Hai contributes in improving and finalizing the system model.

\section{Acknowledgments}

This work was in part supported by the Chong Cheng Chinese School, Phnom Penh, Cambodia. 


\section{References}

[1] C. Huang, W. Jiang, J. Wu, and G. Wang, "Personalized review recommendation based on users' aspect sentiment," $A C M$ Transactions on Internet Technology, vol. 20, no. 4, pp. 1-26, 2020.

[2] C. Chen, B. Liu, S. Wan, P. Qiao, and Q. Pei, "An edge traffic flow detection scheme based on deep learning in an intelligent transportation system," IEEE Transactions on Intelligent Transportation Systems, vol. 22, no. 3, pp. 1840-1852, 2020.

[3] T. Yang, H. Feng, C. Yang, Y. Wang, J. Dong, and M. Xia, "Multivessel computation offloading in maritime mobile edge computing network," IEEE Internet of Things Journal, vol. 6, no. 3, pp. 4063-4073, 2019.

[4] K.-P. Yu, L. Tan, M. Aloqaily, H. Yang, and Y. Jararweh, "Blockchain-enhanced data sharing with traceable and direct revocation in iiot," IEEE Transactions on Industrial Informatics, p. 1, 2021.

[5] S. Wan, J. Hu, C. Chen, A. Jolfaei, S. Mumtaz, and Q. Pei, "Fair-hierarchical scheduling for diversified services in space, air and ground for $6 \mathrm{~g}$-dense internet of things," IEEE Transactions on Network Science and Engineering, 2020.

[6] S. Shahzadi, M. Iqbal, T. Dagiuklas, and Z. Ul-Qayyum, "Multi-access edge computing: open issues, challenges and future perspectives," Journal of Cloud Computing, vol. 6, no. 1, p. 30, 2017.

[7] A. Zhou, S. Wang, S. Wan, and L. Qi, "Lmm: latency-aware micro-service mashup in mobile edge computing environment," Neural Computing and Applications, vol. 32, no. 19, pp. 15411-15425, 2020.

[8] H. Wu, X. Li, and Y. Deng, "Deep learning-driven wireless communication for edge-cloud computing: opportunities and challenges," Journal of Cloud Computing, vol. 9, no. 1, p. 21, 2020.

[9] M. Raja, "Application of cognitive radio and interference cancellation in the l-band based on future air-to-ground communication systems," Digital Communications and Networks, vol. 5, no. 2, pp. 111-120, 2019.

[10] T. Komine and M. Nakagawa, "Fundamental analysis for visible-light communication system using led lights," IEEE transactions on Consumer Electronics, vol. 50, no. 1, pp. 100107, 2004.

[11] L. U. Khan, "Visible light communication: applications, architecture, standardization and research challenges," Digital Communications and Networks, vol. 3, no. 2, pp. 78-88, 2017.

[12] C. Nan, W. Yiguang, and W. Yuanyuan, "Research on multidimensional multiplexing technology of visible light communication," Optics and Optoelecronics Technology, vol. 12, no. 4, pp. 1-6, 2014.

[13] T. Zhao, K. Wright, and X. Zhou, "Lighting up the internet of things with darkvlc," in Proceedings of the 17th International Workshop on Mobile Computing Systems and Applications, HotMobile 2016, pp. 33-38, St. Augustine, FL, USA, 2016.

[14] S. Safavat, N. N. Sapavath, and D. B. Rawat, "Recent advances in mobile edge computing and content caching," Digital Communications and Networks, vol. 6, no. 2, pp. 189-194, 2020.

[15] C. Qing, B. Cai, Q. Yang, J. Wang, and C. Huang, "Elm-based superimposed csi feedback for fdd massive mimo system," IEEE Access, vol. 8, pp. 53408-53418, 2020.

[16] C. Qing, W. Yu, B. Cai, J. Wang, and C. Huang, "Elm-based frame synchronization in burst-mode communication systems with nonlinear distortion," IEEE Wireless Communications Letters, vol. 9, no. 6, pp. 915-919, 2020.

[17] A. Jovicic, J. Li, and T. Richardson, "Visible light communication: opportunities, challenges and the path to market," IEEE Communications Magazine, vol. 51, no. 12, pp. 26-32, 2013.

[18] C. Nan, Led Visible Light Communication Technology, Tsinghua University Press, Beijing, 2014.

[19] A. Pradana, N. Ahmadi, and T. Adiono, "Design and implementation of visible light communication system using pulse width modulation," in Proceedings of the International Conference on Electrical Engineering and Informatics, pp. 25-30, Denpasar, Indonesia, 2015.

[20] C. Yang, W. Liu, X. Li, Q. Yang, and Z. He, "Nyquist-pam-4 transmission using linear dpd and mlse for indoor visible light communications," in Proceedings of the IEEE/CIC International Conference on Communications in China, pp. 22-24, Qingdao, China, 2017.

[21] S. Raza, W. Liu, M. Ahmed et al., "An efficient task offloading scheme in vehicular edge computing," Journal of Cloud Computing, vol. 9, no. 1, p. 28, 2020.

[22] C. Chen, Y. Ding, S. Guo, and Y. Wang, "Davt: an errorbounded vehicle trajectory data representation and compression framework," IEEE Transactions on Vehicular Technology, vol. 69, no. 10, pp. 10606-10618, 2020.

[23] Y. Gong, L. Zhang, R. P. Liu, K. Yu, and G. Srivastava, "Nonlinear mimo for industrial internet of things in cyberphysical systems," IEEE Transactions on Industrial Informatics, vol. 17, no. 8, pp. 5533-5541, 2020.

[24] S. Chen, Y. Wang, and M. Pedram, "A semi-markovian decision process based control method for offloading tasks from mobile devices to the cloud," GLOBECOM, pp. 2885-2890, 2013.

[25] S. Wan, R. Gu, T. Umer, K. Salah, and X. Xu, "Toward offloading internet of vehicles applications in $5 \mathrm{~g}$ networks," IEEE Transactions on Intelligent Transportation Systems, vol. 22, no. 7, pp. 1-9, 2020.

[26] C. Feng, K. Yu, A. K. Bashir et al., "Efficient and secure data sharing for $5 \mathrm{~g}$ flying drones: a blockchain-enabled approach," IEEE Network, vol. 35, no. 1, pp. 130-137, 2021.

[27] S. Wan, Z. Gu, and Q. Ni, "Cognitive computing and wireless communications on the edge for healthcare service robots," Computer Communications, vol. 149, pp. 99-106, 2020.

[28] T. Yang, H. Feng, S. Gao et al., "Two-stage offloading optimization for energy-latency tradeoff with mobile edge computing in maritime Internet of Things," IEEE Internet of Things Journal, vol. 7, no. 7, pp. 5954-5963, 2020.

[29] J. Mills, J. Hu, and G. Min, "Communication-efficient federated learning for wireless edge intelligence in IoT," IEEE Internet of Things Journal, vol. 7, no. 7, pp. 5986-5994, 2020.

[30] T. Komine and M. Nakagawa, "Integrated system of white led visible-light communication and power-line communication," IEEE transactions on Consumer Electronics, vol. 49, no. 1, pp. 71-79, 2003.

[31] H. Le Minh, D. O'Brien, G. Faulkner et al., "100-Mb/s NRZ visible light communications using a postequalized white led," Photonics Technology Letters, vol. 15, no. 1, pp. 1063-1065, 2009.

[32] S. Rajagopal, R. D. Roberts, and S.-K. Lim, "Ieee 802.15.7 visible light communication: modulation schemes and dimming support," IEEE Communications Magazine, vol. 50, no. 3, pp. 72-82, 2012. 
[33] L. Zeng, D. C. O’Brien, H. L. Minh et al., "High data rate multiple input multiple output (mimo) optical wireless communications using white led lighting," IEEE Journal on Selected Areas in Communications, vol. 27, no. 9, pp. 1654-1662, 2009.

[34] Q. Wang, D. Giustiniano, and D. Puccinelli, "Openvlc: software-defined visible light embedded networks," in Proceedings of the 1st ACM MobiCom Workshop on Visible Light Communication Systems, VLCS@MobiCom 2014, pp. 15-20, Maui, Hawaii, USA, 2014.

[35] S. Dimitrov and H. Haas, Principles of LED Light Communications: Towards Networked Li-Fi, Cambridge University Press, Cambridge, 2015.

[36] T. V. Pham, H. L. Minh, and A. T. Pham, "Multi-user visible light communication broadcast channels with zero-forcing precoding," IEEE Transactions on Communications, vol. 65, no. 6, pp. 2509-2521, 2017.

[37] J. M. Kahn and J. R. Barry, "Wireless infrared communications," Proceedings of the IEEE, vol. 85, no. 2, pp. 265-298, 1997.

[38] S. Das, A. Chakraborty, D. Chakraborty, and S. Moshat, "PC to PC data transmission using visible light communication," in Proceedings of the International Conference on Computer Communication and Informatics Coimbatore, pp. 22-27, Coimbatore, India, 2017. 